\title{
Myc is dispensable for cardiac development in the mouse but rescues Mycn- deficient hearts through functional replacement and cell competition
}

${ }^{1}$ Noelia Muñoz-Martín, MSc, ${ }^{1}$ Rocío Sierra, ${ }^{2}$ Thomas Schimmang, PhD, ${ }^{1,}$ Cristina Villa del Campo, PhD, ${ }^{1,{ }^{*}}$ Miguel Torres, PhD

${ }^{1}$ Cardiovascular Development Program, Centro Nacional de Investigaciones Cardiovasculares, CNIC, Madrid

${ }^{2}$ Instituto de Biología y Genética Molecular, Universidad de Valladolid y Consejo

Superior de Investigaciones Científicas

* Authors for Correspondence

Short title: Myc/Mycn regulation of heart development

Co-Corresponding authors:

Cristina Villa del Campo and Miguel Torres

Cardiovascular Development Program, Centro Nacional de Investigaciones

Cardiovasculares, CNIC. Melchor Fernández Almagro 3, Madrid 28029, Spain

Subject Terms: Basic Science Research, Developmental Biology, Myocardial Regeneration 


\begin{abstract}
Myc is considered an essential transcription factor for heart development, but cardiac defects have only been studied in global Myc loss of function models. Here, we eliminated Myc by recombining a Myc floxed allele with the $N k x 2.5 C r e$ driver. We observed no anatomical, cellular or functional alterations in either fetuses or adult cardiac Myc-deficient mice. We re-examined Myc expression during development and found no expression in developing cardiomyocytes. In contrast, we confirmed that Mycn is essential for cardiomyocyte proliferation and cardiogenesis. Mosaic Myc overexpression in a Mycn-deficient background, shows that Myc can replace Mycn function, recovering heart development. We further show that this recovery involves the elimination of Mycn-deficient cells by Cell Competition. Our results indicate that $M y c$ is dispensable during cardiogenesis and adult heart homeostasis and Mycn is exclusively responsible for cardiomyocyte proliferation during heart development. Nonetheless, our results show that Myc can functionally replace Mycn. We also show that cardiomyocytes compete according to their overall Myc+Mycn levels and that Cell Competition eliminates flawed cardiomyocytes, suggesting its relevance as a quality control mechanism in cardiac development.
\end{abstract}

Keywords: Heart development, Transcription factor, proliferation, apoptosis, cell competition 


\section{Introduction}

Myc transcription factors promote cell growth and division, being essential for proliferation in healthy tissues and tumours. Myc proteins belong to the bHLH-domain family and exert their functions mainly by regulating transcription. There are three members of the Myc family of transcription factors in mammals: Myc, Mycn and Mycl. All three transcripts show spatially-restricted patterns during postimplantation embryonic development. Deregulation of these genes has been linked with tumour formation and cell growth.

Myc expression is required for normal embryonic development in mammals, displaying widespread expression from early stages of development, and becoming regionally restricted starting at E7.5. Global Myc KO embryos die between days E9.5 and E10.5, showing defects in heart, pericardium, neural tube and delay or failure of embryo turning ${ }^{1}$. Strong Myc overexpression in transgenic mice enhances myocyte proliferation during heart development, promoting cardiac hyperplasia, which suggested the idea of an essential role of Myc in cardiomyocyte growth and proliferation during development ${ }^{2}$, 3. In contrast, strong Myc overexpression during postnatal life leads to premature cardiomyocyte hypertrophy ${ }^{3,4}$ and heart-specific deletion of Myc prevents hypertrophic growth in response to hemodynamic and pharmacologic stimuli ${ }^{5}$ and cold-induced hypertrophy ${ }^{6}$. Myc mRNA levels however, decrease in correlation with the transition from hyperplastic to hypertrophic growth ${ }^{7}$ and is not expressed in adult cardiomyocytes under normal conditions but becomes strongly activated following hypertrophic stimuli ${ }^{8}$, ${ }^{9}$, which suggests that the physiological function of Myc in postnatal cardiomyocytes is restricted to the hypertrophic response to a challenge. In accordance to this idea, Myc deletion in cardiomyocytes of unchallenged adult mouse hearts does not lead to cardiac function alterations ${ }^{5}$.

Further experiments in a model of moderate overexpression of Myc produced a very different set of results. Mild Myc overexpression in a cellular mosaic fashion does not produce overt phenotypical alterations during embryonic development or adult life, but induces the phenomenon of Cell Competition, by which cells with enhanced anabolism eliminate and replace neighbours without altering tissue homeostasis ${ }^{10,11}$. In cardiacspecific models of Myc mosaic overexpression at moderate levels, Myc-enhanced cardiomyocytes trigger the elimination of neighbouring wild type cardiomyocytes both during development and in the adult heart ${ }^{12,13}$.

The changes induced by Myc moderate overexpression in cardiomyocytes remain within homeostatic limits both during development and in the adult heart ${ }^{12}$. Notably, in these experiments, Myc-enhanced adult hearts are not prone to hypertrophy but display a mild hyperplasic phenotype ${ }^{12}$. The contrast of these results with those obtained by strong overexpression of Myc in transgenic mice ${ }^{3,4}$ suggests that the effects of Myc overexpression critically depend on the levels induced.

While the results obtained in overexpression experiments suggest a role for Myc during cardiomyocyte development, there are no studies reporting cardiac-specific deletion of Myc. Furthermore, the conditional deletion of $M y c$ in the blood/endothelial lineage produces heart defects similar to those observed in the complete Myc elimination ${ }^{14}$ raising the possibility that the cardiac defects observed in the global mutant do not result from a primary function in cardiomyocytes. In contrast, Mycn is essential for cardiomyocyte development in conditional deletion models ${ }^{15}$. Myc and Mycn show high sequence and structure homology and this translates into a highly conserved function, as exemplified by Mycn full rescue of the Myc global knockout in a knock-in replacement mouse model ${ }^{16}$. 
Mycn global mutants die in utero between E10.5 and E11.5, displaying smaller size and hypoplastic heart ${ }^{17-20}$, which is reproduced in a cardiomyocyte-specific deletion of Mycn using a cTnT-Cre driver ${ }^{15}$. Mycn is required for ventricular wall morphogenesis through its role in regulating compact layer cardiomyocyte growth, proliferation and maturation. The defects in heart growth were exclusively due to the reduction in proliferation and not to increased cell death ${ }^{15}$.

Here we studied the role of Myc during heart development, the ability of Myc to rescue Mycn deficiency during cardiogenesis and the involvement of Cell Competition and cardiomyocyte replacement in this rescue. We report the absence of Myc expression or function during cardiac development and the ability of Myc-expressing cardiomyocyte populations to repopulate Mycn-deficient hearts and to rescue Mycn function. Our results indicate that, while Mycn is an essential constitutive component of cardiomyocyte development, Myc is not involved in normal cardiomyocyte formation. Nonetheless, Myc is able to mimic Mycn function, promoting the elimination of Mycndeficient cells to restore a viable heart.

\section{Methods}

\section{Mouse Strains}

iMOS Mouse lines have been previously described ${ }^{10}$. Homozygous iMOS females were mated with males carrying different Cre lines; $W t 1 C r{ }^{21}, N k x 2.5-C^{22}$ to generate embryos. The Mycn floxed allele has been previously described ${ }^{23}$, as has been the Myc-GFP reporter ${ }^{24}$ the Mice were genotyped by PCR. All animal procedures were conducted in accordance with applicable institutional guidelines.

\section{ISH}

WM ISH was performed on E9.5 embryos and E12.5 hearts as described previously, using Myc probe ${ }^{10}$

\section{Confocal microscopy}

Histological sections and whole-mount embryos were imaged with a Nikon A1R confocal microscope using 405, 458, 488, 568 and $633 \mathrm{~nm}$ wavelenghts and 20x/0.75 dry and 40/1.30 oil objectives. Cardiomyocyte nuclei were counted using the cell counter tool of Image J. To estimate cardiomyocyte size, the number of nuclei was divided by the myocardial area calculated using the threshold detection of Image $\mathrm{J}$. Areas occupied by EYFP and ECFP cells and EYFP and ECFP cell number were quantified using the threshold detection and particle analysis tools of the Image $\mathrm{J}$, (NHI, http://rsb.info.nih.gov/ij). To calculate the relative frequency of ECFP cells, the percentage of ECFP cells in each embryo was divided by the average percentage in $i M O S^{W T}$ mosaic. ECFP was scored by subtracting the information of EYFP from the anti-GFP staining which detects both fluorescent proteins.

\section{Adult measurements}

After sacrifice, mice were weighed and hearts were extracted and rinsed in PBS. Hearts were weighed and tibia length of the posterior left leg was measured with a caliber.

\section{Immunofluorescence}

Embryos were fixed overnight at $4^{\circ} \mathrm{C}$ in $2 \%$ paraformaldehyde in PBS and whole-mount 
stained or embedded in gelatin and cryosectioned. Embryonic hearts were fixed in $2 \%$ PFA overnight at $4^{\circ} \mathrm{C}$ and stained in whole-mount. Adult hearts were perfused and fixed in $2 \%$ paraformaldehyde in PBS 24 hours at $4 \stackrel{\circ}{ } \mathrm{C}$ and paraffin-embedded for sectioning. Primary antibodies used were PCM1 (Sigma), a-SMA (Sigma), Myc (Millipore), Living colors Rabbit polyclonal anti GFP antibody (Clontech), c-TnT (Thermo Scientific). Immunofluorescence was performed following standard procedures. TUNEL was performed on heart sections using terminal deoxynucleotidyl transferase (TdT) and biotin-16-2-deoxyuridine-5-triphosphate (Biotin-16-dUTP) (both from Roche), and developed with 647-conjugates Streptavidin (Jackson ImmunoResearch). E9.5 embryos were cleared before whole mount confocal acquisition using ethyl cinnamate as described $\mathrm{in}^{25}$.

\section{Echocardiography study}

Transthoracic echocardiography was performed blinded by an expert operator using a high-frequency ultrasound system (Vevo 2100, Visualsonics Inc., Canada) with a 40$\mathrm{MHz}$ linear probe on a heating platform. Mice were lightly anesthetized with $0.5-2 \%$ isoflurane in oxygen, adjusting the isoflurane to maintain heart rate at $450 \pm 50 \mathrm{bpm}$. A base apex electrocardiogram was continuously monitored. Images were analysed using the Vevo 2100 Workstation software. Parasternal standard, 2D and MM, long and short axis view at the level of the papillary muscles (LAX and SAX view, respectively) were acquired.

\section{Statistical analysis}

Expected versus observed frequencies were compared using the Chi square method.

Adult heart parameters were analyzed with unpaired T-test comparing Wt vs Myc-KO and Wt vs Het separately. Nuclei/myocardium area data was analyzed with Two-way ANOVA. To compare average percentages of ECFP cells between more than two groups, Kruskal-Wallis test was used (assuming non-normal distributions). For comparisons of two groups a Man-Whitney test was used. All comparisons were made using Prism statistical software.

\section{Results and discussion}

\section{Myc is dispensable for heart development and adult heart homeostasis}

To study Myc's role during heart development, we conditionally deleted Myc using the Nkx2.5-Cre strain, which drives widespread Cre-mediated recombination in all cardiac precursors from around E8.022. Embryos resulting from elimination of Myc function in cardiac progenitors (cKO-Myc) were viable and did not display any phenotypic abnormality (Figure 1A). cKO-Myc mice reached adulthood in the expected proportions and presented normal cardiac morphology (Fig 1B, C).

Measurements of heart weight revealed no significant differences in size between cKOMyc homozygous, heterozygous and wild type hearts (Fig 1D). To assess whether Myc loss had any effect on cardiomyocyte size or number, we estimated the density of cardiomyocyte nuclei in histological sections from different regions of the left ventricle (LV) (Fig 1E). The density of cardiomyocyte nuclei was similar between cKO-Myc homozygous, heterozygous and control hearts (Fig 1F). These data, together with the consistency in heart size, suggests that neither cardiomyocyte size nor number are changed by the loss of Myc expression. 
To functionally assess cKO-Myc hearts, we performed echocardiographic assays on 10 -week adult mice. No significant differences were found between groups in ejection fraction and fractional shortening parameters, indicating that the function of cKO-Myc hearts is not affected by the loss of Myc (Fig 1G). Overall, cKO-Myc hearts display normal morphology and function and therefore our data indicate that Myc is dispensable for heart formation and adult heart homeostasis.

\section{Myc is not detectably expressed in developing cardiomyocytes}

The results obtained could be explained by the lack of Myc function during cardiomyocyte development or by the compensation of a putative Myc function by Mycn. Myc RNA expression has been reported by Northern blot in mid-gestation samples from whole myocardium ${ }^{2,7}$ and Myc protein expression has been reported by Western blot from whole adult myocardium ${ }^{5}$. Here, we performed in situ analyses to determine which cells express Myc during myocardial development. We first performed Myc mRNA in situ hybridization (ISH) detection during embryogenesis ${ }^{10}$. At embryonic day 9.5 (E9.5) Myc displayed expression in the neural tube, branchial arches, cephalic regions and other non-cardiac tissues (Fig 2A), which is in agreement with previous reports $^{26}$. At this stage Myc mRNA was not detected in the heart tube, while within the cardiogenic region, expression was seen in the proepicardium (Fig 2A). Analysis at later stages showed weak Myc mRNA detection in the distal outflow tract (OFT) and subepicardium at E12.5 (Fig 2B). These results were contradictory with our previous characterization of Myc protein distribution using anti-Myc antibodies in immunofluorescence, in which a clear signal was detected in cardiomyocytes at $\mathrm{E} 10.5^{12}$. To resolve this contradiction, we repeated the immunofluorescence comparing wild type and cKO-Myc hearts at E10.5 (Figure 2 C, D). Detection of Myc expression in wild type embryos clearly identified a nuclear signal in cardiomyocytes (Fig 2C). This signal remained unchanged in cKO-Myc hearts (Fig 2D). This result contrasts with the observation that this antibody has been validated for endogenous Myc detection in the E6.5 mouse epiblast ${ }^{10}$. These results suggest that Myc antibody cross-reacts with other members of the family in cardiomyocytes, most likely Mycn, whose role and expression in cardiomyocyte development is well established, while it is not expressed in the E6.5 epiblast $^{15,18}$.

To further confirm Myc expression in the developing heart, we took advantage of a Myc-GFP knock-in reporter line in which endogenous Myc protein expression is reported by the green fluorescent protein (GFP) fused to the endogenous Myc open reading frame ${ }^{24}$. In accordance to our ISH results, Myc-GFP expression at E9.5 was strongly detected in the branchial arches (Fig 2E) and in the proepicardium; however, no Myc expression was detected in the myocardium at this stage.

We conclude that Myc does not play a role in cardiomyocyte development because it is not expressed in this lineage and thus it does not act in redundancy with Mycn.

\section{Forced Myc expression in a mosaic fashion is sufficient to rescue cKO-Mycn cardiac defects}

A relevant question is whether the different effects reported for Myc overexpression in cardiomyocytes result from Myc mimicking Mycn function by Myc. As mentioned above, $M y c n$ can replace Myc functions when knocked-in to the Myc locus ${ }^{16}$. Here we addressed whether Myc could replace Mycn function in the developing heart. To test this, we used Myc overexpression from the Cre-inducible Rosa26R-iMOS mosaic system. The $i M O S^{T 1 M y c}$ allele allows the induction of mild overexpression of Myc in a 
cellular mosaic fashion ${ }^{10}$ (Fig $3 \mathrm{~A}$ ). In this mosaic model, $75 \%$ of recombined cells overexpress Myc and are reported by EYFP expression, while $25 \%$ do not overexpress Myc and are reported by the ECFP expression. We established crosses to obtain embryos simultaneously deleted for a conditional Mycn allele (cKO-Mycn) and activated for iMOS ${ }^{T 1 M y c}$ with the Nkx2.5-Cre driver (Fig 3B). Embryos in which Mycn has been deleted but the iMOS mosaic is not activated are not viable past E10.5-E11.5 (Fig 3B), in accordance with the phenotype previously reported for Mycn deletion in cardiomyocytes $^{15}$. Similarly, iMOS ${ }^{\text {T1Myc }}$ activation on a wild type or Mycn-heterozygous background (iMOS-Myc) does not produce any phenotypic alteration ${ }^{12}$. Interestingly, cKO-Mycn littermates in which the $i M O S^{t 1 M y c}$ mosaic has been activated (cKO-Mycn; iMOS-Myc) were viable and indistinguishable from iMOS-Myc littermates (Fig. 3B). Histological analysis and study of the contribution of the cells recombined by Nkx2.5Cre at E13.5 showed normal contribution of cardiac progenitors to the heart and no morphological alterations in either cKO-Mycn or Mycn-Wt hearts with iMOS-Myc overexpression (Fig. 3C). These results indicate that mosaic overexpression of one dose of Myc, driven by the endogenous Rosa26 promoter, is enough to functionally replace the loss of Mycn expression during heart development.

\section{Cell Competition contributes to the rescue of Mycn-deficient hearts by stimulating the replacement of deficient cells}

The complete phenotypic rescue of $c K O-M y c n$ hearts suggested that, in addition to cell-autonomous replacement of Mycn function by Myc, some cell non-autonomous mechanism should operate to either eliminate or rescue the $25 \%$ of cells that do not activate Myc. To understand which of these mechanisms is at work, we determined the proportion of ECFP and EYFP cardiomyocyte populations in different genetic configurations. Control iMOS mosaics expressing only the fluorescent proteins over a wild type background produce a $25-75 \%$ distribution of ECFP and EYFP cardiomyocytes when activated by the Nkx2.5-Cre driver (iMOS-Wt) (Figure 4A,D) and the same experiment performed with the $i M O S^{T 1 M y C}$ mosaic reduces the cell population that does not overexpress Myc (ECFP cells) to $60 \%$ of the original, due to cell competition (Fig 4D) ${ }^{12}$. When the same experiment was performed over a cKO-Mycn heterozygous background, the proportion of ECFP cells observed was about $50 \%$ in E10.5 hearts (MycnHet ; iMOS-Myc) (Fig 4B, D) whereas in a background homozygous for cKO-Nmyc (cKO-Mycn ; iMOS-Myc), the proportion of ECFP cells dropped to $15 \%$ (Fig 4C, D). This elimination was further exacerbated in the cKO-Mycn homozygous background with progression of development, showing a proportion of only $4.5 \%$ of the original pool of ECFP cells at E13.5 (Fig 4F, D). These results suggest that not only the cell-autonomous replacement of Mycn by Myc, but also a replacement of Mycndeficient cells by iMOS-Myc overexpressing cells contributes to the rescue of cKOMycn hearts.

We next explored the possibility that the elimination of this cell population takes place by Cell Competition. Elimination of Mycn-deficient cells when confronted with Mycoverexpressing cells was much more efficient than elimination of wild type or Mycnheterozygous cells, which would fit a scenario in which both Myc and Mycn act additively to determine cardiomyocyte competition ability. An alternative view would be that Mycn-deficient cells are not actively eliminated but just diluted out due to their limited ability to proliferate. To discriminate between these possibilities, given that Mycn-deficient cardiomyocytes do not autonomously display an increased apoptotic rate $^{15}$ and that apoptosis is a hallmark of Myc-induced cell competition in the developing mouse heart, we performed TUNEL analysis. 
As previously reported, in $i M O S^{t 1 M y c}$ mosaics induced over a wild type background the EYFP-Myc cell population shows nearly undetectable apoptosis, while the ECFP-WT cardiomyocyte population shows close to $6 \%$ apoptotic rate (Figure $4 \mathrm{E})^{12}$. In contrast, in iMOS $^{\text {T1MyC }}$ mosaics induced over the cKO-Mycn background, while the apoptotic rate was also very low in EYFP-Myc cells, the apoptotic rate of the ECFP Mycn-KO cells increased over $50 \%$ (Figure 4F, G). These results indicate that confrontation with Mycrescued cells produces a strong selective apoptotic elimination of Mycn-KO cells, otherwise viable in a homotypic environment ${ }^{15}$.

Taken together, our results show that Myc is not required for heart development and is not detectably expressed in developing cardiomyocytes. In the context of previous evidences from adult heart endogenous Myc expression and function analyse ${ }^{2,5,7}$, it is concluded that Myc expression and role in heart physiology is restricted to stress responses during adult life, while Mycn fully assumes the constitutive roles of the family during cardiogenesis. In addition, we show that Myc can replace Mycn in its functions during cardiomyocyte development and that Cell Competition contributes to rescuing heart function by stimulating the elimination of defective cells. This demonstration adds to previous evidence indicating the great plasticity of the developing heart to adapt to the progressive loss of up to $50 \%$ of its cardiomyocyte population by compensatory proliferation of the healthy population ${ }^{27}$. In contrast to this previously reported model, in which the unhealthy cardiomyocyte population is autonomously predisposed to cell death $^{27}$, in the model presented here the unhealthy cell population is not prone to cell death when in isolation but undergoes massive cell death by confrontation with a Mycrescued cell population. These results suggest an endogenous role for cell competition in the correction of contingent defects that may appear in cardiomyocytes during development.

\section{Acknowlegdments}

We thank members of the Torres group for stimulating discussions and suggestions. We thank members of the microscopy and histopathology CNIC units, led by Valeria Caiolfa and Antonio de Molina-Iracheta, respectively, for excellent support and sample processing. We also thank the CNIC Advanced Imaging Unit and the CNIC Animal Facility personnel for their excellent work and support.

\section{Sources of funding}

This work is supported by a Leducq Foundation grant "Redox Regulation of Cardiomyocyte Renewal" and by grants BFU2015-71519-P and RD16/0011/0019 (ISCIII) from the Spanish Ministry of Science, Innovation and Universities. The CNIC is supported by the Ministerio de Ciencia, Innovación y Universidades and the Pro CNIC Foundation, and is a Severo Ochoa Center of Excellence (SEV-2015-0505).

\section{Disclosures}

None 


\section{References}

1. Davis AC, Wims M, Spotts GD, Hann SR, Bradley A. A null c-myc mutation causes lethality before 10.5 days of gestation in homozygotes and reduced fertility in heterozygous female mice. Genes \& development. 1993;7:671-682

2. Jackson T, Allard MF, Sreenan CM, Doss LK, Bishop SP, Swain JL. The c-myc proto-oncogene regulates cardiac development in transgenic mice. Molecular and cellular biology. 1990;10:3709-3716

3. Machida N, Brissie N, Sreenan C, Bishop SP. Inhibition of cardiac myocyte division in c-myc transgenic mice. Journal of molecular and cellular cardiology. 1997;29:1895-1902

4. Xiao G, Mao S, Baumgarten G, Serrano J, Jordan MC, Roos KP, Fishbein MC, MacLellan WR. Inducible activation of c-myc in adult myocardium in vivo provokes cardiac myocyte hypertrophy and reactivation of DNA synthesis. Circulation Research. 2001;89:1122-1129

5. Zhong W, Mao S, Tobis S, Angelis E, Jordan MC, Roos KP, Fishbein MC, de Alboran IM, MacLellan WR. Hypertrophic growth in cardiac myocytes is mediated by myc through a cyclin d2-dependent pathway. The EMBO journal. 2006;25:3869-3879

6. Bello Roufai M, Li H, Sun Z. Heart-specific inhibition of protooncogene c-myc attenuates cold-induced cardiac hypertrophy. Gene therapy. 2007;14:14061416

7. Schneider MD, Payne PA, Ueno H, Perryman MB, Roberts R. Dissociated expression of c-myc and a fos-related competence gene during cardiac myogenesis. Molecular and cellular biology. 1986;6:4140-4143

8. Pollack PS, Houser SR, Budjak R, Goldman B. C-myc gene expression is localized to the myocyte following hemodynamic overload in vivo. Journal of cellular biochemistry. 1994;54:78-84

9. Izumo S, Nadal-Ginard B, Mahdavi V. Protooncogene induction and reprogramming of cardiac gene expression produced by pressure overload. Proceedings of the National Academy of Sciences of the United States of America. 1988;85:339-343

10. Claveria C, Giovinazzo G, Sierra R, Torres M. Myc-driven endogenous cell competition in the early mammalian embryo. Nature. 2013;500:39-44

11. Claveria C, Torres M. Cell competition: Mechanisms and physiological roles. Annual review of cell and developmental biology. 2016;32:411-439

12. Villa Del Campo C, Claveria C, Sierra R, Torres M. Cell competition promotes phenotypically silent cardiomyocyte replacement in the mammalian heart. Cell reports. 2014;8:1741-1751

13. Villa Del Campo C, Lioux G, Carmona R, Sierra R, Munoz-Chapuli R, Claveria $\mathrm{C}$, Torres $\mathrm{M}$. Myc overexpression enhances of epicardial contribution to the developing heart and promotes extensive expansion of the cardiomyocyte population. Scientific reports. 2016;6:35366

14. He C, Hu H, Braren R, Fong SY, Trumpp A, Carlson TR, Wang RA. C-myc in the hematopoietic lineage is crucial for its angiogenic function in the mouse embryo. Development. 2008;135:2467-2477

15. Harmelink C, Peng Y, DeBenedittis P, Chen H, Shou W, Jiao K. Myocardial mycn is essential for mouse ventricular wall morphogenesis. Developmental biology. 2013;373:53-63

16. Malynn BA, de Alboran IM, O'Hagan RC, Bronson R, Davidson L, DePinho RA, Alt FW. N-myc can functionally replace c-myc in murine development, cellular growth, and differentiation. Genes \& development. 2000;14:1390-1399 
17. Charron J, Malynn BA, Fisher P, Stewart V, Jeannotte L, Goff SP, Robertson EJ, Alt FW. Embryonic lethality in mice homozygous for a targeted disruption of the n-myc gene. Genes \& development. 1992;6:2248-2257

18. Moens CB, Stanton BR, Parada LF, Rossant J. Defects in heart and lung development in compound heterozygotes for two different targeted mutations at the n-myc locus. Development. 1993;119:485-499

19. Stanton BR, Perkins AS, Tessarollo L, Sassoon DA, Parada LF. Loss of n-myc function results in embryonic lethality and failure of the epithelial component of the embryo to develop. Genes \& development. 1992;6:2235-2247

20. Sawai S, Shimono A, Wakamatsu Y, Palmes C, Hanaoka K, Kondoh H. Defects of embryonic organogenesis resulting from targeted disruption of the n-myc gene in the mouse. Development. 1993;117:1445-1455

21. Wessels A, van den Hoff MJ, Adamo RF, Phelps AL, Lockhart MM, Sauls K, Briggs LE, Norris RA, van Wijk B, Perez-Pomares JM, Dettman RW, Burch JB. Epicardially derived fibroblasts preferentially contribute to the parietal leaflets of the atrioventricular valves in the murine heart. Developmental biology. 2012;366:111-124

22. Stanley EG, Biben C, Elefanty A, Barnett L, Koentgen F, Robb L, Harvey RP. Efficient cre-mediated deletion in cardiac progenitor cells conferred by a 3'utrires-cre allele of the homeobox gene nkx2-5. The International journal of developmental biology. 2002;46:431-439

23. Knoepfler PS, Cheng PF, Eisenman RN. N-myc is essential during neurogenesis for the rapid expansion of progenitor cell populations and the inhibition of neuronal differentiation. Genes \& development. 2002;16:2699-2712

24. Huang CY, Bredemeyer AL, Walker LM, Bassing CH, Sleckman BP. Dynamic regulation of c-myc proto-oncogene expression during lymphocyte development revealed by a gfp-c-myc knock-in mouse. European journal of immunology. 2008;38:342-349

25. Klingberg A, Hasenberg A, Ludwig-Portugall I, Medyukhina A, Mann L, Brenzel A, Engel DR, Figge MT, Kurts C, Gunzer M. Fully automated evaluation of total glomerular number and capillary tuft size in nephritic kidneys using lightsheet microscopy. Journal of the American Society of Nephrology : JASN. 2017;28:452-459

26. Uslu VV, Petretich M, Ruf S, Langenfeld K, Fonseca NA, Marioni JC, Spitz F. Long-range enhancers regulating myc expression are required for normal facial morphogenesis. Nature genetics. 2014;46:753-758

27. Drenckhahn JD, Schwarz QP, Gray S, Laskowski A, Kiriazis H, Ming Z, Harvey RP, Du XJ, Thorburn DR, Cox TC. Compensatory growth of healthy cardiac cells in the presence of diseased cells restores tissue homeostasis during heart development. Developmental cell. 2008;15:521-533 
A

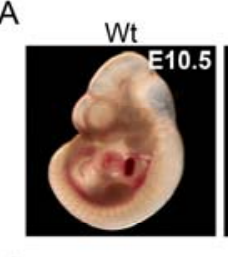

C

\begin{tabular}{|c|c|c|c|}
\cline { 3 - 4 } & \multicolumn{2}{c|}{ Frequency } \\
\hline Genotype & Adult mice & Observed & Expected \\
\hline Wt & $\mathbf{2 0}$ & $\mathbf{0 . 2 6 6}$ & $\mathbf{0 . 2 5}$ \\
\hline cHet-Myc & 38 & $\mathbf{0 . 5 0 6}$ & $\mathbf{0 . 5 0}$ \\
\hline cKO-Myc & $\mathbf{1 8}$ & $\mathbf{0 . 2 4 0}$ & $\mathbf{0 . 2 5}$ \\
\hline \multicolumn{2}{|c}{} & \multicolumn{2}{c}{$\mathrm{p}=0.948$} \\
\hline
\end{tabular}
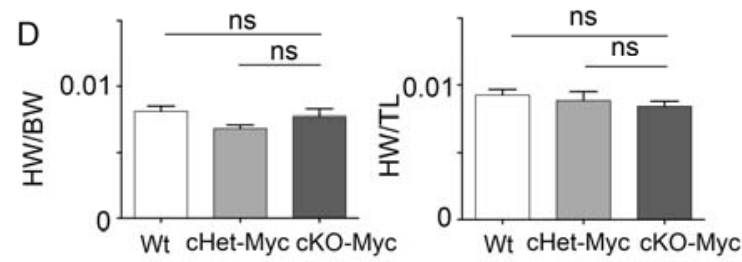

$\mathrm{E}$
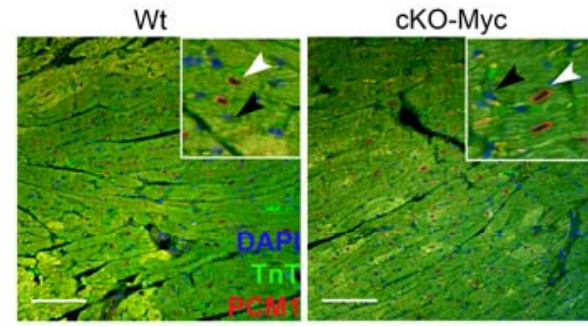

$\mathrm{F}$

$\square$ Wt $\square$ cHet-Myc $\quad$ =cKO-Myc
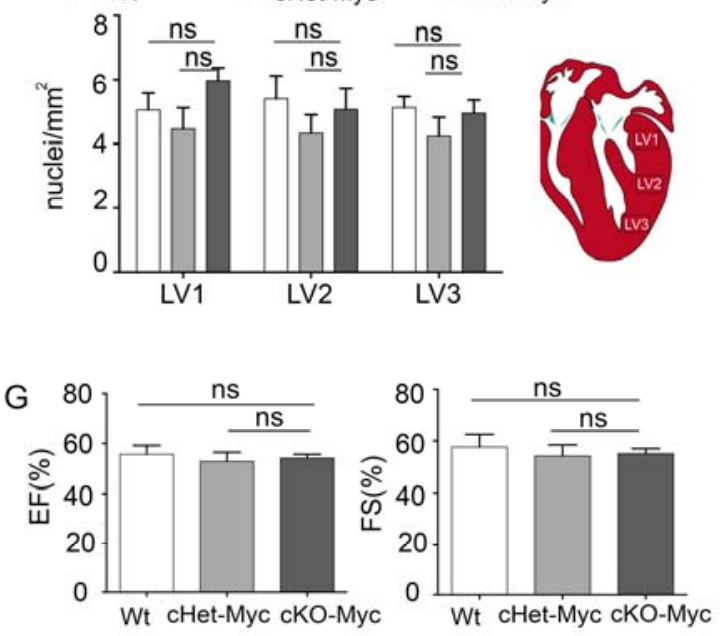

Figure 1. Myc is dispensable for heart development and adult heart in homeostasis. A. Whole mount wild type E10.5 embryo (left) and cKO-Myc E10.5 embryo (right). B. Whole mount wild type adult heart (left) and cKO-Myc adult heart (right). C. Table depicting the observed and expected frequencies of adult mice from the different genotypes. D. Graphs depicting the heart/body weight and heart/tibia length ratios in 10-week-old animals. E. Confocal images from sections of adult hearts stained with anti-PCM1 (red) and anti-TnT (green). F. Graph showing the quantification of $\mathrm{CM}$ nuclei per area in three different regions of the left ventricle. Location of the regions within the left ventricle is identified in the schematic as LV1, LV2 and LV3 Bar: 100um Data in $\mathbf{D}, \mathbf{F}, \mathbf{G}$ are means \pm SEM; ns: $p>0,05 . \mathrm{N}=3-8$ mice/condition 


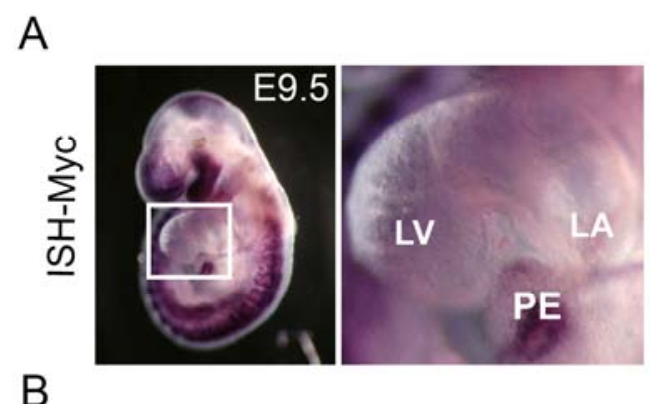

B
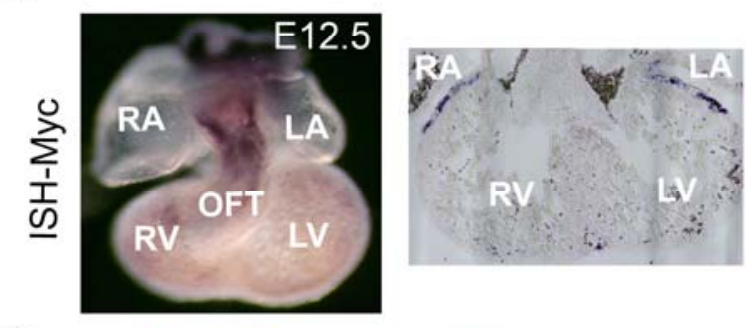

C

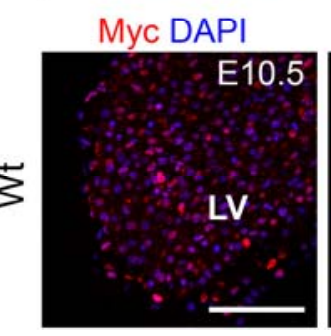

Myc
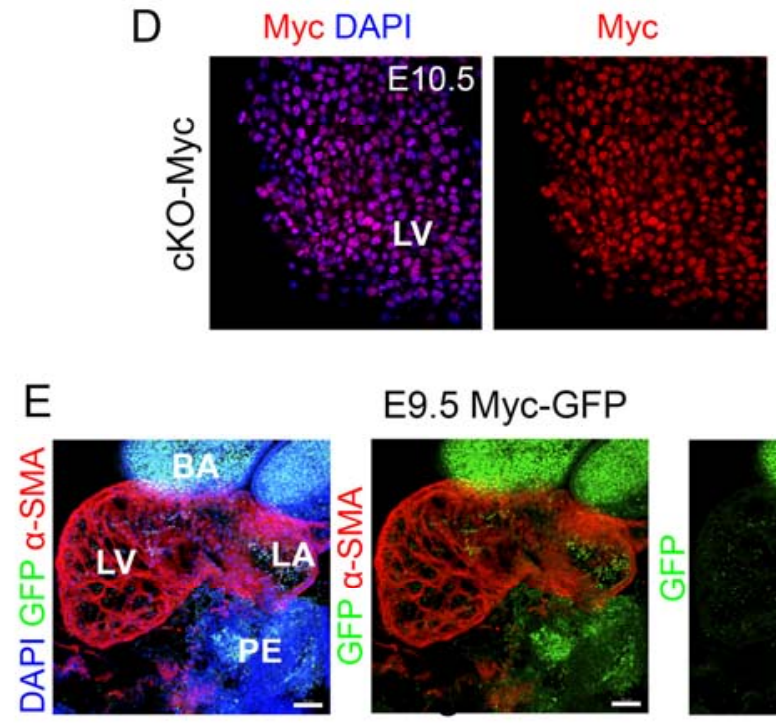

E9.5 Myc-GFP
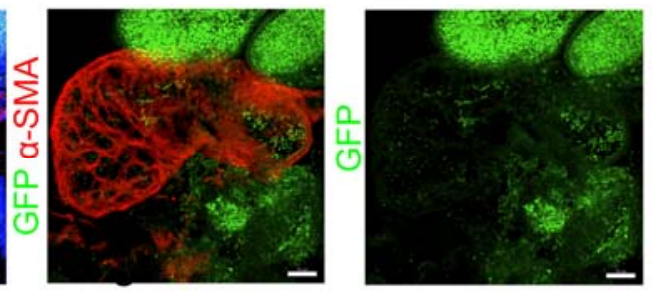

Figure 2. Myc is not widely expressed in cardiomyocytes during heart development. A. Whole mount in situ hybridization in E9.5 WT embryo for Myc, showing no expression in the heart (box in A). B. Whole mount in situ hybridization of a E12.5 WT embryonic heart for Myc. C-D. Confocal section of a whole E10.5 heart showing staining for Myc and DAPI (left) and Myc (right) for Wt (C) and cKO-Myc (D) embryo. E. Confocal section of a whole mount E9.5 Myc-GFP embryo showing GFP Myc expression and a-SMA. Bar, $50 \mu \mathrm{m}$ in C, D and 70 in E. LV: Left ventricle, RV: Right ventricle, RA: Right atria, LA: Left atria, OFT: Outflow tract, BA: Branchial arches, PE: Proepicardium 

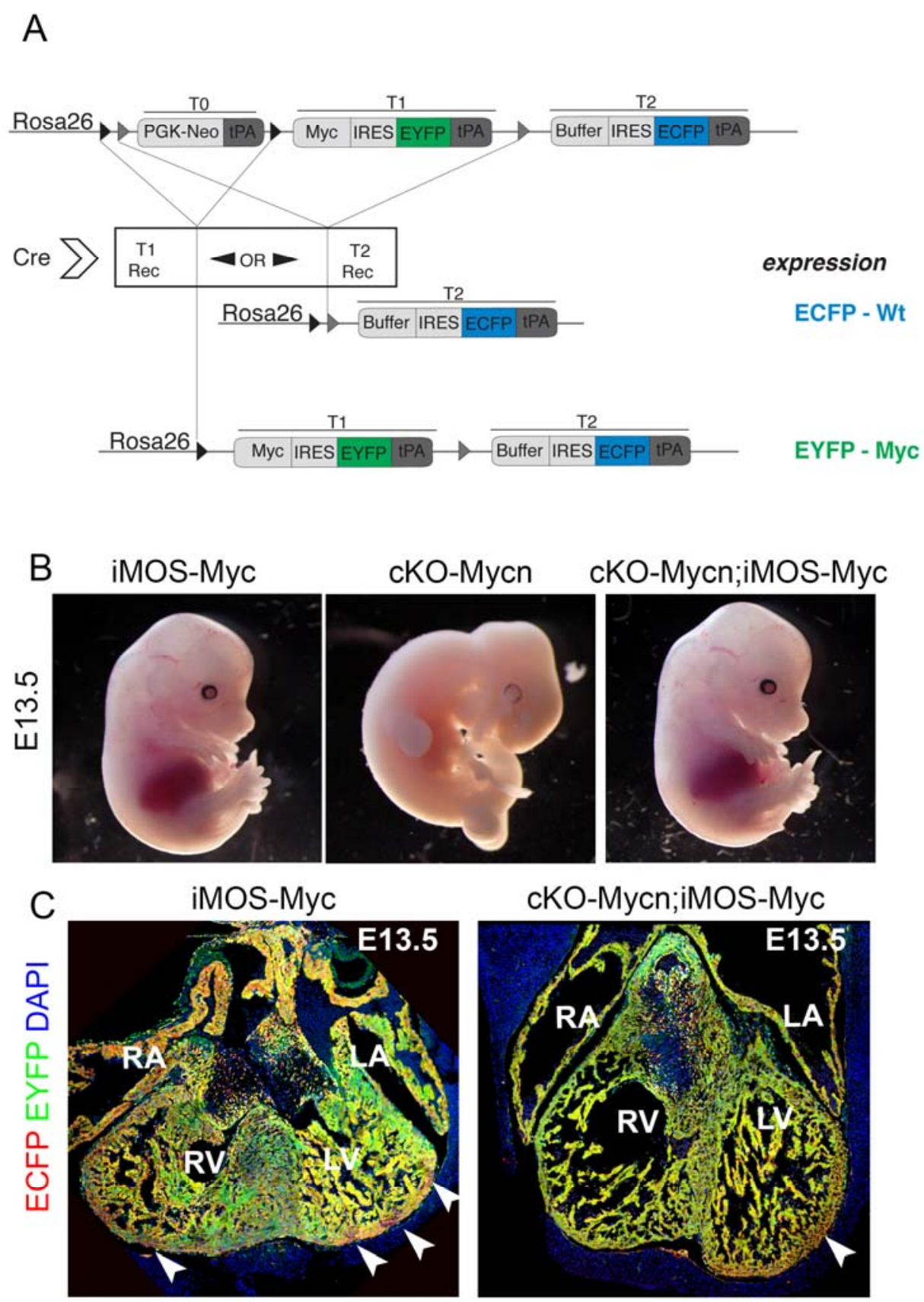

Figure 3. Myc expression in a mosaic fashion is sufficient to rescue Mycn embryonic lethal phenotype. A. Schematic of the iMOS construction. If TO cassette is excised, T1 is expressed (EYFP-Myc). If T2 recombination takes place both T0 and $\mathrm{T} 1$ are excised leading to the expression of T2 (ECFP-Wt). This gives rise to two labeled cell populations at random under the control of Rosa26 promoter. B. Whole mount image of a E13.5 iMOS-Myc embryo (left), cKO-Myc embryo (middle) and cKOMycn ; iMOS-Myc (right). C. Confocal section of a E13.5 heart from a iMOS-Myc embryo, showing EYFP-Myc and ECFP-wt cell populations (left) and cKO-Mycn ; iMOS-Myc embryo, showing EYFP-Myc and ECFP-wt cell populations (right). Arrowheads point to ECFP positive cells. LV: Left ventricle, RV: Right ventricle, RA: Right atria, LA: Left atria. ( $\mathrm{N}=3$ cKO-Mycn ; iMOS-Myc, $\mathrm{N}=3$ cKO-Mycn, $\mathrm{N}=3$ iMOSMyc in Mycn heterozygous or wild type backgrounds). 


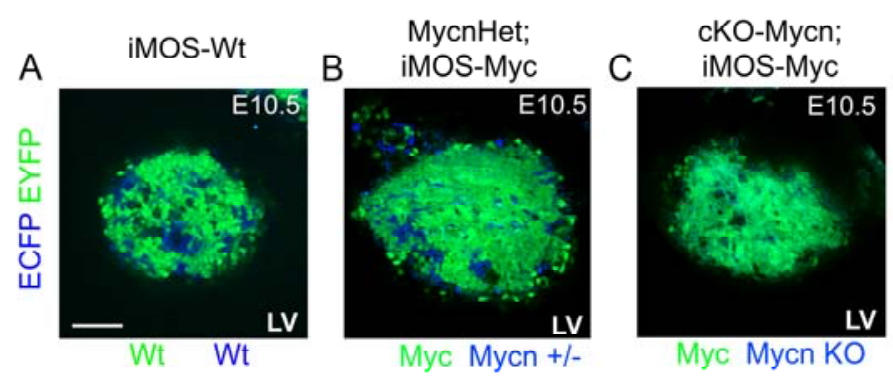

D
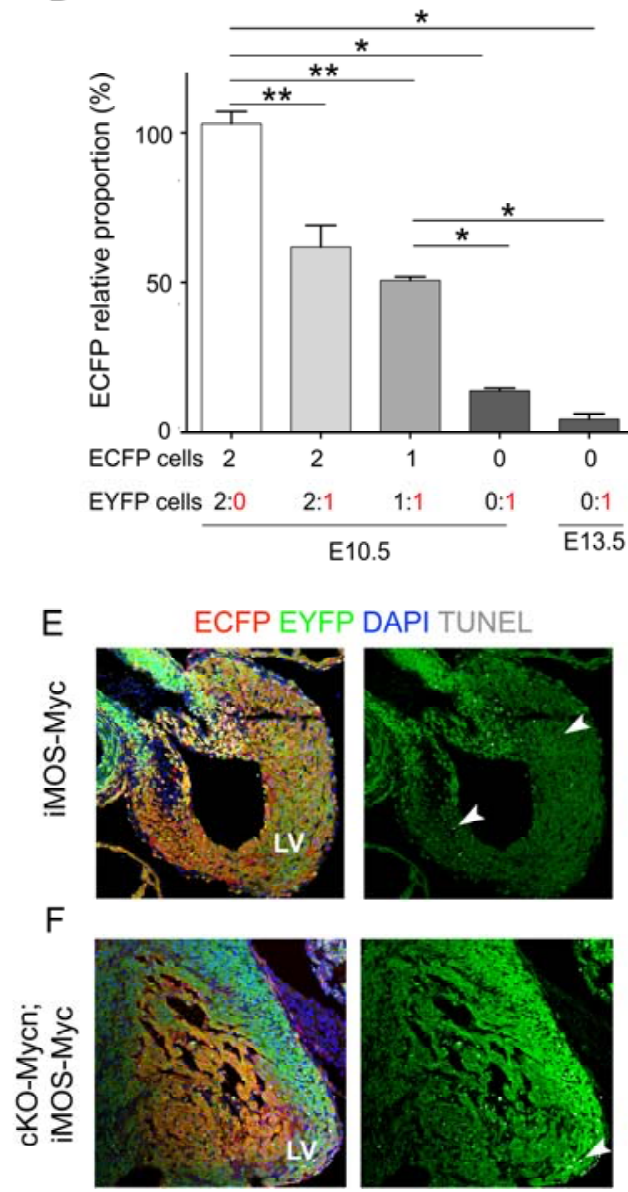

Mycn endogenous alleles Myc extra copies

G

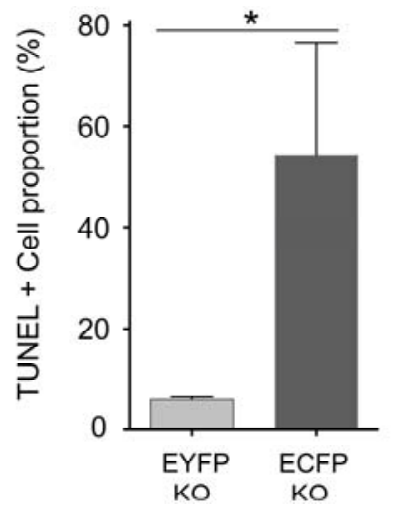

Figure 4: Myc and Mycn interplay can trigger a competitive process in cardiac progenitors, displacing those cells with lower relative Myc/Mycn levels, actively eliminated by cell competition. A-C Confocal sections from E10.5 hearts from iMOSWt (A), Mycn-Het ; iMOS-Myc (B) and cKO-Mycn ; iMOS-Myc (C) showing an overlay of EYFP and ECFP. D. Percentage of ECFP+ cells in hearts of the iMOS-Myc (MYC) mosaics in the three different Mycn backgrounds relative to that observed in the iMOSWT(WT) mosaics, normalized to $100 \%$. E. Confocal images of E13.5 heart sections of iMOS-Myc hearts showing ECFP and EYFP cell populations and TUNEL positive cells. F. Confocal images of E13.5 heart sections of cKO-Mycn ; iMOS-Myc hearts showing ECFP and EYFP cell populations and TUNEL positive cells. G, Graph showing the relative percentage of TUNEL+ cells in EYFP and ECFP cell populations in CKOMycn;iMOS-Myc embryonic hearts. Bar $50 \mu \mathrm{m}$. LV: Left ventricle. Data in $\mathbf{D}$ are means $\pm S E M ;{ }^{*} p<0,05 ;{ }^{* *} p<0,01$. Data in $G$ are means $\pm S E M$. Arrowheads in $E$ and $F$ point to TUNEL positive ECFP cells. 